\title{
Um Algoritmo Quântico para um Problema de Distância Estatística*
}

\author{
Henrique Hepp ${ }^{1}$, Murilo V. G. da Silva ${ }^{1}$, Leandro M. Zatesko ${ }^{2}$ \\ ${ }^{1}$ Departamento de Informática, Universidade Federal do Paraná \\ ${ }^{2}$ Departamento Acadêmico de Informática, Universidade Tecnológica Federal do Paraná \\ \{hhepp, murilo\}@inf.ufpr.br, zatesko@utfpr.edu.br
}

\begin{abstract}
In the Statistical Distance to Uniform Distribution (SDU) problem, the aim is to compare a probability distribution over all n-bit strings with the uniform distribution. In this paper, we deal with the restriction of SDU in which the probabilities of the $2^{n} / 2$ first strings (under the usual lexicographic ordering) are never smaller than the $2^{n} / 2$ last strings. We prove that this restriction admits a polynomial-time quantum algorithm.
\end{abstract}

Resumo. No problema Distância Estatística para Distribuição Uniforme (SDU), o objetivo é comparar uma distribuição de probabilidade sobre as strings de $n$ bits com a distribuição uniforme. Neste trabalho, lidamos com a restrição de $S D U$ em que as probabilidades das $2^{n} / 2$ primeiras strings (sob a ordenação lexicográfica usual) nunca são menores que as das $2^{n} / 2$ últimas. Provamos que esta restrição admite um algoritmo quântico polinomial.

\section{Introdução}

Distância Estatística para Distribuição Uniforme (SDU), conforme definido a seguir, é um problema de promessa em que precisamos comparar uma distribuição de probabilidade $X$ com a distribuição uniforme $U_{n}$, ambas sobre todas as strings com $n$ bits, perguntando-nos o quão próximo a distância estatística $\Delta\left(X, U_{n}\right)$ está de 0 ou de 1 . Este problema é completo para NISZK, a classe dos problemas que admitem um protocolo não-interativo de conhecimento zero estatístico [Goldreich et al. 1999].

\section{$\underline{\text { SDU }}$}

Dados:

um inteiro não-negativo $n$ e um circuito booleano $C$ de tamanho polinomial em $n$ com $n$ bits de saída, o qual codifica uma distribuição de probabilidade $X$ sobre todas as strings com $n$ bits;

decidir entre: instância positiva: $\Delta\left(X, U_{n}\right)<1 / n$;

instância negativa: $\Delta\left(X, U_{n}\right)>1-1 / n$;

prometido que vale um dos casos.

Não se sabe se SDU está em BQP, a classe dos problemas que admitem algoritmos quânticos polinomiais.

Distância Estatística entre Distribuição Biparticionada e Distribuição Uniforme $(B S D U)$ é o problema SDU restrito a distribuições de probabilidade biparticionadas. No

\footnotetext{
*Realizado com apoio parcial de CAPES (código 001) e CNPQ (428941/2016-8).
} 
nosso contexto, dizemos que uma distribuição de probabilidade $Y$ sobre todas as strings com $n$ bits, codificada por um circuito booleano $C$ com $n$ bits de saída, é biparticionada se, para qualquer string $x$ dentre as $2^{n} / 2$ primeiras (sob a ordenação lexicográfica usual $00 \cdots 00, \ldots, 11 \cdots 11$ ) e qualquer string $y$ dentre as $2^{n} / 2$ últimas, a probabilidade de se obter $x$ como saída de $C$ é não menor que a probabilidade de se obter $y$.

Neste trabalho, provamos que BSDU está em BQP, exibindo um algoritmo quântico polinomial para o problema. Em contrapartida, parece não haver uma maneira direta de se colocar o problema em BPP usando técnicas análogas, uma vez que nosso algoritmo trata todas as $2^{n}$ strings em sobreposição quântica.

Este trabalho está organizado do seguinte modo: na Seção 2, apresentamos alguns preliminares e, na Seção 3, a demonstração de que BSDU está em BQP.

\section{Preliminares 1}

Sendo o estado de um sistema quântico um vetor unitário de números complexos representado por $|\psi\rangle$, seu transposto conjugado é representado por $\langle\psi|$. O produto interno entre dois vetores $\psi_{1}, \psi_{2}$ é representado por $\left\langle\psi_{1} \mid \psi_{2}\right\rangle$. O produto diádico entre $\psi_{1}$ e $\psi_{2}$ é representado por $\left|\psi_{1}\right\rangle\left\langle\psi_{2}\right|$. Um estado $|\psi\rangle$ em um espaço $\mathbb{C}^{N}$ pode ser descrito pela combinação linear de $N$ estados linearmente independentes $|\psi\rangle=\alpha_{1}|1\rangle+\alpha_{2}|2\rangle+\cdots+$ $\alpha_{N}|N\rangle$, para $\alpha_{1}, \cdots \alpha_{N} \in \mathbb{C}$, dizemos que esses $N$ estados estão em sobreposição. Um qubit é um estado quântico $|\psi\rangle=\alpha|0\rangle+\beta|1\rangle$, para $\alpha, \beta \in \mathbb{C}$ e $|\alpha|^{2}+|\beta|^{2}=1$. Um estado quântico de $n$ qubits é um produto tensorial entre $n$ qubits.

Dado o estado $|\Psi\rangle=\sum_{i=0}^{2^{n}-1} \alpha_{i}|i\rangle$, podemos fazer uma medição quântica de $|\Psi\rangle$ com relação à base $\left\{|0\rangle,|1\rangle, \cdots,\left|2^{n}-1\right\rangle\right\}$, obtendo um dos estados $|i\rangle$ com probabilidade $\left|\alpha_{i}\right|^{2}$. Um circuito quântico é um grafo dirigido acíclico em que cada vértice é ou uma transformação unitária (representada por uma matriz unitária), ou uma medição agindo sobre um qubit. O tamanho do circuito é o número de vértices do grafo.

Sendo $\left|\psi_{1}\right\rangle, \ldots,\left|\psi_{k}\right\rangle$ estados quânticos de $n$ qubits, um estado misto de $n$ qubits é representado por um operador de densidade, ou matriz de densidade, que é a matriz de $\mathbb{C}^{2^{n} \times 2^{n}}$ definida por $\rho:=\sum_{i=1}^{k} p_{i}\left|\psi_{i}\right\rangle\left\langle\psi_{i}\right|$, sendo $p_{i}$ a probabilidade clássica de ocorrer o estado $\psi_{i}$, de modo que $\sum_{i=1}^{k} p_{i}=1$. Se $k=1$, o estado $\rho$ é chamado de estado puro. $\mathrm{O}$ traço de uma matriz de densidade é sempre igual a 1 e os valores de sua diagonal são reais e não-negativos. Caso $\rho$ seja uma matriz diagonal, representa uma distribuição de probabilidade, com cada uma das $2^{n}$ entradas da diagonal sendo a probabilidade referente a cada uma das strings de $n$ bits. A matriz de densidade $1 / 2^{n}$ é conhecida como matriz de densidade maximalmente mista e sua diagonal corresponde à distribuição uniforme.

Dada uma matriz de densidade de $n$ qubits, as matrizes de densidade reduzidas referentes a $m<n$ qubits podem ser obtidas pelo descarte de $n-m$ qubits por meio da operação de traço parcial (trace out). Dada uma matriz de densidade $\rho_{A B}$ que pode ser decomposta nas bases $\left\{\left|a_{i}\right\rangle\right\}$ e $\left\{\left|b_{i}\right\rangle\right\}$ como $\rho_{A B}=\sum_{i j k \ell} c_{i j k \ell}\left|a_{i}\right\rangle\left\langle a_{j}|\otimes| b_{k}\right\rangle\left\langle b_{\ell}\right|$, a matriz de densidade reduzida da parte $A$, obtida ao se fazer o traço parcial da parte $B$ do sistema, é dada pela expressão $\operatorname{tr}_{B} \rho_{A B}:=\sum_{i j k \ell} c_{i j k \ell}\left|a_{i}\right\rangle\left\langle a_{j}\right|\left\langle b_{\ell} \mid b_{k}\right\rangle$.

Definimos a distância de traço entre dois operadores de densidade $\rho$ e $\sigma$ como

\footnotetext{
${ }^{1}$ Referimos o leitor a [Nielsen and Chuang 2011] para mais detalhes sobre Computação Quântica.
} 
$\|\rho-\sigma\|_{\text {tr }}:=\frac{1}{2} \sum_{i}\left|\lambda_{i}\right|$, sendo $\left\{\lambda_{i}\right\}$ os autovalores de $\rho-\sigma$. Se $\rho$ e $\sigma$ são matrizes diagonais, $\|\rho-\sigma\|_{\text {tr }}$ é igual à distância estatística entre as distribuições que $\rho$ e $\sigma$ representam.

Distância de Traço para Um Qubit $((\alpha, \beta)-1 Q S D)$, para $0 \leq \alpha<\beta \leq 1$ é o problema de promessa em que, dados dois circuitos quânticos $Q_{1}$ e $Q_{2}$, com $m>1$ qubits de entrada e 1 qubit de saída cada, os quais devolvem respectivamente os estados mistos $\rho$ e $\sigma$ ao receberem $|0\rangle^{\otimes m}$, decidir entre $\|\rho-\sigma\|_{\text {tr }} \leq \alpha$ e $\|\rho-\sigma\|_{\text {tr }} \geq \beta$, prometido que vale um dos casos. É bem conhecido que este problema pode ser resolvido em tempo $O(\operatorname{poly}(m))$ quântico sempre que $\alpha$ e $\beta$ não estão próximos demais.

Proposição 1. Para $0 \leq \alpha<\beta \leq 1 \operatorname{com} 1 /(\beta-\alpha)^{2}=\operatorname{poly}(m)$, o problema $(\alpha, \beta)$ $1 Q S D$ está em BQP.2.

\section{Um algoritmo quântico para BSDU}

Recordemos, da definição do problema SDU, que recebemos um circuito booleano clássico $C$ de tamanho polinomial em $n$ que codifica uma distribuição de probabilidade $X$ sobre todas as strings com $n$ bits. Conforme trabalhos sobre classes quânticas de conhecimento zero [Watrous 2002, Kobayashi 2003], é possível, a partir de $C$, obter em tempo polinomial clássico um circuito quântico $Q$ com $n$ qubits de saída e $m>n$ de entrada tal que, recebendo $|0\rangle^{\otimes m}$, devolve o estado misto cuja matriz de densidade $\rho$ é a matriz diagonal das probabilidades das $2^{n}$ strings. Deste modo, supomos, para nosso problema BSDU, que a entrada não é o circuito clássico $C$, mas o circuito quântico $Q$.

Mostramos que, quando os primeiros $2^{n} / 2$ da diagonal de $\rho$ não são maiores ou iguais aos outros $2^{n} / 2$ elementos, pode-se resolver BSDU em tempo polinomial quântico ao se considerar apenas o primeiro qubit. Note-se que para SDU em geral esta estratégia não funciona, pois podemos ter o caso de $\rho$ representar uma distribuição suficientemente distante da uniforme, i.e. $\left\|\rho-\mathbb{1} / 2^{n}\right\|_{\operatorname{tr}}>1-1 / n$, e ainda assim a cada qubit individualmente corresponder a matriz de densidade maximalmente mista.

Teorema 2. BSDU está em BQP.

Prova. Supomos sem perda de generalidade que $n>3$, pois, caso contrário, poderíamos decidir entre $\Delta\left(X, U_{n}\right)<1 / n$ e $\Delta\left(X, U_{n}\right)>1-1 / n$ em tempo $O(1)$ clássico. Seja $\rho$ a matriz de densidade devolvida pelo circuito quântico $Q$, conforme discutimos. Sejam $\alpha=1 / n, \beta=1-1 / n, N=2^{n}$, e $\sigma$ a matriz de densidade reduzida do primeiro qubit obtida ao se fazer o traço parcial dos demais qubits de $\rho$. Vamos mostrar que: (i) se $\|\rho-\mathbb{1} / N\|_{\operatorname{tr}}<\alpha$, então $\|\sigma-\mathbb{1} / 2\|_{\operatorname{tr}}<\alpha$; (ii) se $\|\rho-\mathbb{1} / N\|_{\text {tr }}>\beta$, então $\|\sigma-\mathbb{1} / 2\|_{\text {tr }}>\beta / 2$.

Observe que $\sigma=\left(\sum_{i=1}^{N / 2} \rho_{i i}\right)|0\rangle\left\langle 0\left|+\left(\sum_{i=N / 2+1}^{N} \rho_{i i}\right)\right| 1\right\rangle\langle 1|$. Considerando a definição do problema BSDU e que $\rho$ e $\sigma$ são matrizes diagonais, fazendo $\delta_{k}:=\rho_{k k}-1 / N$ para todo $k$, temos $\delta_{i} \geq \delta_{j}$ para todo $i \leq N / 2$ e todo $j>N / 2$ e:

$$
\begin{aligned}
\|\rho-\mathbb{1} / N\|_{\text {tr }} & =\frac{1}{2}\left(\left|\delta_{1}\right|+\cdots+\left|\delta_{N}\right|\right) \\
\|\sigma-\mathbb{1} / 2\|_{\text {tr }} & =\frac{1}{2}\left(\left|\delta_{1}+\delta_{2}+\cdots+\delta_{N / 2}\right|+\left|\delta_{N / 2+1}+\cdots+\delta_{N}\right|\right) .
\end{aligned}
$$

\footnotetext{
${ }^{2}$ Para uma discussão mais detalhada sobre a qual este resultado pode ser inferido, ver e.g. [Montanaro and de Wolf 2016, p. 44, Sec. 4.2, dois primeiros parágrafos].
} 
(i) Por desigualdade triangular, $\|\sigma-\mathbb{1} / 2\|_{\operatorname{tr}}<\alpha$ segue imediatamente da promessa $\|\rho-\mathbb{1} / N\|_{\text {tr }}<\alpha$.

(ii) Como o traço de $\rho$ é igual a 1 e todos os elementos de $\rho$ são reais não-negativos, temos $\sum_{\delta_{i}>0} \delta_{i}=\sum_{\delta_{i}<0}\left|\delta_{i}\right|>\beta$, e $-1 / N \leq \delta_{i} \leq 1-1 / N$ para todo $i$. Observe que, de $\sum_{\delta_{i}<0}\left|\delta_{i}\right|>\beta$, segue que $x:=\left|\left\{\delta_{i}: \delta_{i}>0\right\}\right|>0$ e $\left|\left\{\delta_{i}: \delta_{i}<0\right\}\right|=N-x>N / 2$. Portanto, como $\delta_{i} \geq \delta_{j}$ para todo $i \leq N / 2$ e todo $j>N / 2$, a soma $\mid \delta_{1}+\delta_{2}+\cdots+$ $\delta_{N / 2}|+| \delta_{N / 2+1}+\ldots+\delta_{N} \mid$ é minimizada quando todos os $N-x$ valores em $\left\{\left|\delta_{i}\right|: \delta_{i}<0\right\}$ são iguais a

$$
\frac{\sum_{\delta_{i}<0}\left|\delta_{i}\right|}{N-x}>\frac{\beta}{N-x} .
$$

Assim,

$$
\begin{aligned}
\|\sigma-\mathbb{1} / 2\|_{\operatorname{tr}} & =\frac{1}{2}\left(\left|\delta_{1}+\delta_{2}+\cdots+\delta_{N / 2}\right|+\left|\delta_{N / 2+1}+\ldots+\delta_{N}\right|\right) \\
& >\frac{1}{2}\left(\beta-\left(\frac{N}{2}-x\right) \frac{\beta}{N-x}+\frac{N}{2}\left(\frac{\beta}{N-x}\right)\right) \\
& \geq \frac{\beta}{2}\left(\frac{N}{N-x}\right) \geq \frac{\beta}{2}\left(\frac{N}{N-1}\right)>\frac{\beta}{2}
\end{aligned}
$$

Como consequência, o problema BSDU reduz-se a $(1 / n, 1 / 2-1 /(2 n))-1 \mathrm{QSD}$; com isto, o algoritmo polinomial quântico para BSDU segue da Proposição 1 .

\section{Referências}

Goldreich, O., Sahai, A., and Vadhan, S. (1999). Can statistical zero knowledge be made non-interactive? or On the relationship of SZK and NISZK. In Annual International Cryptology Conference, pages 467-484.

Kobayashi, H. (2003). Non-interactive quantum perfect and statistical zero-knowledge. In International Symposium on Algorithms and Computation, pages 178-188.

Montanaro, A. and de Wolf, R. (2016). A survey of quantum property testing. Theory Comput., (7):1-81.

Nielsen, M. A. and Chuang, I. L. (2011). Quantum Computation and Quantum Information: 10th Anniversary Edition. Cambridge University Press, 10th edition.

Watrous, J. (2002). Limits on the power of quantum statistical zero-knowledge. In The 43rd Annual IEEE Symposium on Foundations of Computer Science, 2002. Proceedings., pages 459-468. IEEE. 